\title{
The Application of MRF Based-on Chaos-PSO Optimization in Depth Information Estimation
}

\author{
Xiangjin Zeng ${ }^{1, a}$,Cheng Lu $^{2}$ \\ ${ }^{1}$ The key Lab. of Intelligence robotic of Hubei Province, Wuhan 430074, China \\ ${ }^{2}$ School of Computer Science and Engineering, WIT , Wuhan 430074, China \\ axjzeng21@sohu.com
}

Keywords : signal vision; markov random field;depth estimation; chaos-PSO optimization

\begin{abstract}
For depth information estimation of vision image, a blur parameter model of defocus image based on Markov random field (MRF) has been present. It converts problem of depth estimation into optimization problem. An improved chaos-PSO Algorithm has been applied to complete optimization problem, which prevents that the result gets into local optimization. The experiments and simulations prove that the model and algorithm are efficiency.
\end{abstract}

\section{Introduction}

The vision is a stress approach which manipulation robotic obtains external information. As we know, the vision with single CCD camera can only obtain 2D information in task space. In order to operate the object in 3D space, It is a exigent problem we encountered that obtains the depth of the object. To obtain the depth of manipulation from 2D plane image is a research hot topic in robotic vision. Many peoples[3][4][5][6] have done something to deal with it. Usually, There are several methods to obtain the depth of object. One is that employs stereo vision to restore the 3D image from the $2 \mathrm{D}$ image, which the algorithm of stereo match is very difficult. The second method we focused on is that computes blur characterization of image in the frequency domain to obtain depth. Third, As a representative, Pentland[7]has presented the method of Depth from Defocus based the blur image, which computes image object's depth from two blur images in same scene, with changing the inner and external parameter of CCD camera. Pentland's method depends on CCD camera system model and precise system parameter.

Since the change in the depth of a scene is usually gradual, the blur parameter tends to have local dependencies. Hence, we are motivated to model the blur parameter as a MRF[1][9]. A defocus image blur parameter model base MRF has been presented in this paper. It converts the depth problem into energy function optimization problem. Then, applies an improved chaos-PSO algorithm to optimization energy function, which prevents that the result gets into local optimization. Experiments and simulations confirm the efficiency of model and algorithm. This paper is organized as follows. Section two gives CCD camera imaging model of camera vision defocus image, Section three constructs a blur parameter model of defocus image based on MRF, The Chaos-PSO algorithm and it's implementation is presented in section four. Section five carries out experiments and simulations and conclusion is given in section six.

\section{CCD Camera Imaging Model}

According to CCD imaging principle, we can give formula as shown in (1)

$$
\frac{1}{f}=\frac{1}{u 0}+\frac{1}{\nu 0}
$$

Where $f$ is the focus length, $u 0$ is the distances from object to camera, and $\nu 0$ is the distances from image focus point to camera lens. When the distances from object to camera lens is $u 0$ and the distances from camera plane to camera lens is 10 , we can obtain a clear image. If changes the 
distances from camera plane to camera lens, a blur image can be seen in CCD camera plane. When keeps a constant for camera parameter, It can be given the relationship between image defocus radius and the depth of image as shown in (2)

$$
u=\frac{f i 0}{10-f-F r_{b}}
$$

For formula (2), $\mathrm{F}$ is the $\mathrm{F}$ number of CCD lens. $r_{b}$ is the defocus radius of image and $\mathrm{u}$ is the depth of the image. Therefore, There is a corresponding function relationship between the distances from CCD lens to object and the defocus radius of blur image, which can be used to obtain the depth of the image. According to formula (2), the positive or negative of distances $u$ depends on if the focus image locates fore or back in image plane. We restrict that the distances of object is higher than the distances of image. The formula (2) can be converted into (3)

$$
r_{b}=\frac{f v 0}{F}\left(\frac{1}{f}-\frac{1}{v 0}-\frac{1}{u}\right)
$$

For two captured image with different focus length setting, we have formula (4)

$$
r_{b}^{i}=\frac{f_{i} v_{i}}{F}\left(\frac{1}{f_{i}}-\frac{1}{v_{i}}-\frac{1}{u}\right)
$$

For defocus image, the blur parameter is $\rho$ and is given by $\rho=\beta r_{b}$, With giving value $\mathrm{i}=12$ and eliminating $u$, the relationship of two defocus image's blur parameter is shown as (5)

$$
\rho^{1}=m \rho^{2}+n
$$

Since the blur parameter $\rho^{i}$ at location $(\mathrm{x}, \mathrm{y})$ is related to the depth of the scene, we can construct a model of the blue parameter based on MRF, meaning that the depth of the scene can be obtained indirectly.

\section{Constructing The Blur Parameter Model Based MRF}

Let $\mathrm{X}$ denotes the random fields corresponding to the blur parameter $\rho^{i}$, $\mathrm{X}$ can be modeled by MRF. Namely, it shows as in (6)

$$
P(X=x)=\frac{1}{Z} e^{-U(x)}
$$

If $Y_{1}, Y_{2}$ denote the random fields corresponding to the two observed images, the posterior probability can be expressed as (7)

$$
P\left(X \mid Y_{1}, Y_{2}\right)=\frac{P\left(Y_{1}, Y_{2} \mid X\right) P(X)}{P\left(Y_{1}, Y_{2}\right)}
$$

Where $P\left(Y_{1}, Y_{2}\right)$ is a constant and $P(X)$ is the previous probability of the blur parameter. $P\left(X \mid Y_{1}, Y_{2}\right)$ is the posterior probability of the initial image, with knowing $\mathrm{Y}$ value .So, according to Bayes rules, the depth restoration of the defocus image can be converted into the problem that seeks the estimation of the original image when the posterior probability is maximization.

\section{Implementation}

A. The previous probability computation

Given $\mathrm{X}$ as the blur parameter of the defocus image, Let thinks $\mathrm{X}$ as a MRF, the previous probability $P(X)$ can be used Gibbs distribution to descript. 


$$
P(X)=\frac{1}{z}\left[e^{-\sum_{x \in C} V_{c}(x)}\right]
$$

For given the observed images y1, y2, $P\left(Y_{1}=y 1, Y_{2}=y 2\right)$ is a constant. Hence, the probability $P\left(Y_{1}=y 1, Y_{2}=y 2 \mid X\right)$ can be descript as Gaussian distribution and be shown as (9)

$$
P\left(Y_{1}=y 1, Y_{2}=y 2 \mid X\right)=e^{-\sum^{2} \frac{1}{2 \sigma^{2}}\left(y_{1}-\mu_{4}\right)^{2}-\sum_{s} \frac{1}{2 \sigma^{2}}\left(y_{2}-\mu_{2}\right)^{2}}
$$

Then, formula (7) can be converted into (10)

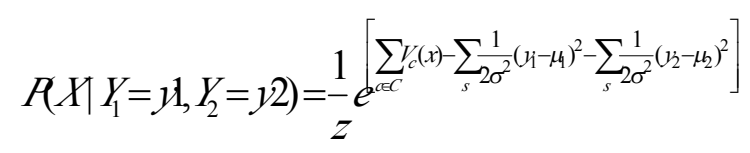

Based on the observed image $\mathrm{y} 1, \mathrm{y} 2$, the problem of depth estimation is to find the estimation $x$ of $\mathrm{X}$, which can computes the depth indirectly.

Base on discussion above, the posterior probability $P\left(X \mid Y_{1}, Y_{2}\right)$ about the original image can be converted into the optimization problem as shown in formula

$$
\min \left[-\sum_{c \in C} V_{c}(x)+\sum_{s} \frac{1}{2 \sigma^{2}}\left(y_{1}-\mu_{1}\right)^{2}+\sum_{s} \frac{1}{2 \sigma^{2}}\left(y_{2}-\mu_{2}\right)^{2}\right]
$$

B Chaos-particle swarm optimization algorithm

Particle Swarm Optimization (PSO) proposed by Kennedy and Eberhart, is a new evolutionary algorithm which the inspiration comes from the social behavior of birds and fish stocks. The advantages of PSO is the extremum seeking simplicity and effectiveness, and has been successfully applied on the neural network, SVM, optimization. However, PSO optimization may lead to local minima due to the premature convergence. In order to overcome the local minimum of the PSO, this paper uses chaos search to increase the diversity of the particles. The chaotic search all states in the search space generated approximation optimization solutions neighbor to keep the solution diversity. Chaos and PSO integration can prevent precocious optimization process.

PSO involves two important parameters, namely, individual extreme ${ }^{P_{e}}$ and global extreme G. The algorithm of the update speed $v$ and position of is as follows

$$
\begin{aligned}
& v_{i}(k+1)=\omega_{i} v_{i}(k)+c_{1}^{\prime}\left[P_{e}-P_{i}(k)\right] \\
& +c_{2}{ }_{2}\left[G-P_{i}(k)\right] \\
& P_{i}(k+1)=P_{i}(k)+v_{i}(k+1)
\end{aligned}
$$

In (11), ${ }^{\omega_{i}}$ is the inertia weight, ${ }^{r_{1}}$ and ${ }^{r_{2}}$ is random coefficients from [0,1], ${ }^{c_{1}}$ and ${ }^{c_{2}}$ is speeding constant. Quadratic optimization chaos algorithm overcomes the premature of standard particle swarm optimization, and Logistic equation is the most used Chaos Algorithm, the mapping expression

$$
P_{i}(k+1)=\mu P_{i}(k)\left(1-P_{i}(k)\right)
$$

${ }^{\mu}$ is a control parameter. The chaos optimization process is as follows: First, the flight position $P$ is mapped to $[0,1]$ chaotic variables: 


$$
Q_{i}(k)=\left(P_{i}(k)-a_{i}\right) / b_{i}
$$

The ${ }^{a_{i}}$ and $b_{i}$ is given parameter, and then calculate the chaotic behavior:

$$
Q_{i}(k+1)=\mu Q_{i}(k)\left(1-Q_{i}(k)\right)
$$

After a few iterations, we can find optimal value $f^{*}$ of the best $Q_{i}$, the second carrier is used to map the chaotic back to the original space:

$$
P_{i}(k)=a_{i}+b_{i} Q_{i}(k)
$$

In this process, chaos algorithm can find effectively a reasonable global extreme G to help PSO jump out of the local optimal solution.

Logistic convergence speed is very slow, and it is not efficient. Therefore, we can improve the ability of chaos search by the use of the Henon mapping. Henon map is as follows:

$$
\begin{aligned}
& P_{i}(k+1)=1-c P_{i}(k)^{2}+d Q_{i}(k) \\
& Q_{i}(k+1)=P_{i}(k)
\end{aligned}
$$

\section{Experiments And Simulations}

We can obtain the multi defocus images from a single camera in the experimental system, which adjust the focal length of camera. Then, we calculate the absolute depth of the scenery from the two defocus images

Firstly, we construct the restoration model of the vision defocus image based on MRF the same as (14). Presumes that the observed images are y1, y2 and defines $Y$ as the restoration image. $Y$ is thought as a MRF. Then, we can restore the defocus image similarly as (14). During manipulation experiments, presumes the camera work distances (the distances from object lens to clear imaging plane.) $\mathrm{u} 0=60 \mathrm{~mm}$, and gives that micro-move platform zero point corresponding effector tip position as original point in coordinate. Then, we revise vision system in order to locate original point in clear imaging plane.

Fig.1 and Fig.2 show the initial defocus image of gripper driven by piezoelectricity with different camera setting. Fig. 3 gives the restoration image of gripper driven by piezoelectricity.

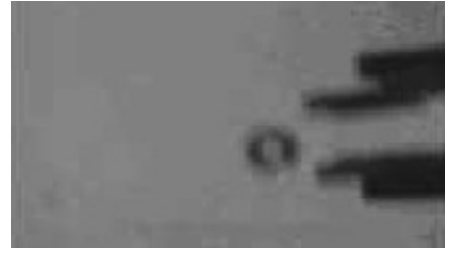

Fig1. The initial image of defocus image gripper driven by piezoelectricity

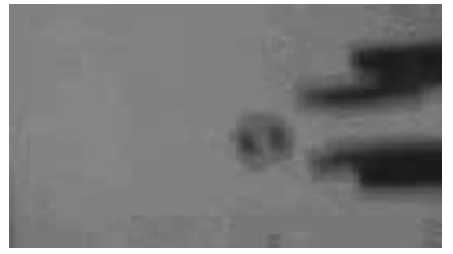

Fig2. The initial image of defocus image gripper driven by piezoelectricity with different

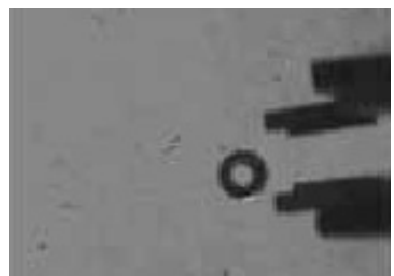

Fig 3. The restoration image of defocus image of gripper driven by piezoelectricity camera parameters settings

Secondly, We give the performance of estimation of depth method. The image is captured by Panasonic CCD camera with focus length of $4 \mathrm{~cm}$. The lens aperture was kept constant at an $\mathrm{f}$ number of 5. Two defocused images of the scene are taken for two different focusing ranges of $70 \mathrm{~cm}$ and $95 \mathrm{~cm}$, which the nearest and the farthest points were at a distance of $70 \mathrm{~cm}$ and $95 \mathrm{~cm}$ from the camera to the object.

We demonstrate the performance of the method in estimating blur parameter and recovering the depth. The method of Subbarao[6] is employed to obtain initial estimates of $\rho$. We choose 
$\rho_{i, j}{ }^{2}=0.6 \rho_{i, j}{ }^{1}$ and the number of level for the blur parameter is 25. Figs. 4,5,6,7 show the experiment results. The original defocus image with blur $\rho^{1}$ and blur $\rho^{2}$ is shown in Fig4 and Fig5, respectively. Fig6 shows the estimated value of depth obtained using DFD, which the initial point is given randomly(according to equation of the blur parameter.). Correspondingly, the estimated value of the depth employed the proposed method that the initial point is chose using LSE is shown in Fig8. From this figures, Compared with DFD that the initial point is given randomly, we note that the planar nature of the variation in the depth of the scene is better brought by the proposed method.

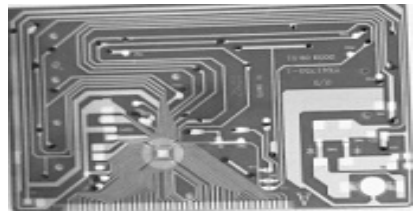

Fig4. The original defocus image of print circuit broad with the blur $\rho^{1}$

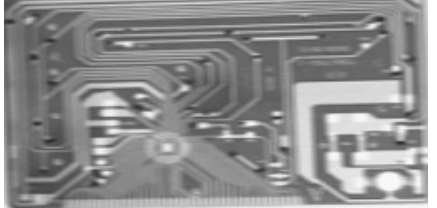

Fig5. The original defocus

image of print circuit broad with the blur $\rho^{2}$

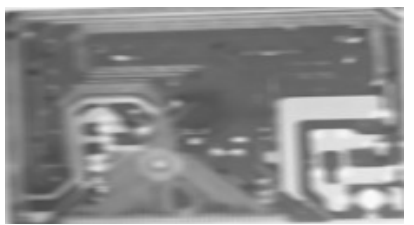

Fig6. The estimated value of

the depth obtained using DFD

with random the initial point

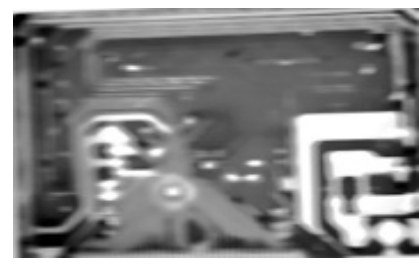

Fig7. The estimated value of the depth obtained using proposed method

we can see from the experimental results that the test system extracts the depth information of objects in high precision at close range. In the test, it has a large relatively error between the actual depth and the target depth from the experiment test when the target object is the farther from the camera. To the camera that has the fixed pixel size and the fixed imaging plane size, the smaller imaged on the image plane, the fewer the useful information of pixels they contain. Then, it affects that the stereo matching accuracy is deteriorated. So, the error of extracted target depth information and the relative error have also become large. Table 1 shows the result of obtaining depth information that use the proposed method .

Table 1 Depth extraction tests

\begin{tabular}{l|l|l|l}
\hline Target & $\begin{array}{c}\text { Target actual } \\
\text { depth }(\mathrm{mm})\end{array}$ & $\begin{array}{c}\text { Target } \\
\text { measurement } \\
\text { depth }(\mathrm{mm})\end{array}$ & $\begin{array}{c}\text { Relative } \\
\text { error }(\%)\end{array}$ \\
\hline 1 & 200 & 200.20 & 0.1 \\
\hline 2 & 250 & 250.37 & 0.14 \\
\hline 3 & 300 & 300.79 & 0.26 \\
\hline 4 & 350 & 351.01 & 0.28 \\
\hline 5 & 400 & 400.97 & 0.24 \\
\hline 6 & 450 & 451.12 & 0.25 \\
\hline 8 & 500 & 502.60 & 0.5 \\
\hline
\end{tabular}

\section{Conclusion}

The depth estimation in micromanipulation tasks is a key technology in microscope vision system. For the depth estimation of microscope vision image, This paper presents a blur parameter model of the defocus image based on MRF. It converts problem of depth estimation into optimization problem. 
An improved Iterated Conditional Modes Algorithm has been applied to complete optimization problem, which prevents that performance result gets into local optimization. The experiments and simulations prove that the model and algorithm is efficiency. It provides the probability that finishes visual servoing control in 3D space.

\section{References}

[1] Lu Mingjun, Wang Ruisheng, "A Markov random field method in computer vision," Journal of Electron Science, vol. 22, pp. 1028-1037, 2000.

[2]Vinay P. Namboodiri, Subhasis Chaudhuri, "On defocus, diffusion and depth estimation," Pattern Recognition Letters, vol 28, pp.311-319,2007.

[3]Du ming TSAI, Chin tun lin, “A moment preserving approach for depth from defocus," Pattern Recognition, vol 31, No.5, pp. 551-560,1998.

[4]F.Deschenes, D.Ziou, P.Fuchs, "An unified approach for a simultaneous and cooperative estimation of defocus blur and spatial shifts," Image and Vision Computing , pp. 35-57, 2004.

[5]Pentland A, Darrell T, Turk M, Huang W, "A simple, real-time range camera," Proceedings of the IEEE Conference on Computer Visionand Pattern Recognition, pp. 256-261, 1989.

[6]Subbarao M, "Parallel depth recovery by changing camera parameters," Proceedings of the IEEE Conference on Computer Vision ,pp.149-155,1988.

[7]A.N. Rajagopalan and S.Chaudhuri, "An MRF model based approach to simultaneous recovery of depth and restoration form defocused images," IEEE transactions on pattern analysis and machine intelligence, vol 21, No.7, pp. 577-589, 1999.

[8]Jaehyun Park, Ludwik Kurz, "Image enhancement using the modified ICM method," IEEE transactions on image processing, vol 5, No.5, pp. 765-771, 1996.

[9]Aamir Saeed Malik, Tae Sun Choi, "Consideration of illumination effects and optimization of window size for accurate calculation of depth map for 3D shape recovery," pattern recognition, vol 40, pp. 154-170, 2007.

[10]A.N.Rajagopalan and S.Chaudhuri, "A variational approach to recovering depth from defocused images," IEEE transactions on pattern analysis and machine intelligence, vol 19, No.10, pp. 1158-1164, 1997.

[11]Ovidiu Ghita, Paul F.Whelan, "A video rate range sensor based on depth from defocus," Optics \& Laser Technology, pp. 167-176, 2001.

[12]Jong Kae Fwu, Petar M.Djurio, Member, IEEE, "Unsupervised vector image segmentation by a trss structure - ICM algorithm,”, IEEE transtions on medical imaging, vol 15, No.6, pp.871-880, 1996.

[13]Wei Ding. A new Method for image noise removal using chaos-PSO and nonlinear ICA [J]. Procedia Engineering. 24(2011)111-115..

\section{Acknowledgment}

This work is supported by the National Nature Science Foundation of China under Grant 11101318 and the key Lab of image processing and intelligence control (201104) and the Science Foundation of. WIT and the young Foundation of. WIT. ( Q201206) and the key Lab of intelligence robotic of Hubei Province. (HBIR200903, HBIR200905). 